\title{
Singularity Handling on Puma in Operational Space Formulation
}

\author{
Denny Oetomo, Marcelo Ang Jr. \\ National University of Singapore \\ Singapore \\ d_oetomo@yahoo.com \\ mpeangh@nus.edu.sg \\ Ser Yong Lim \\ Gintic Institute of Manufacturing Technology, Singapore \\ sylim@gintic.gov.sg
}

\begin{abstract}
A solution to the singularity problem is presented from the approach of the operational space formulation which involves both motion and force control. A brief summary of the Operational Space Formulation and how singularity presents a problem is explained. The inverse of the Jacobian at singular configuration is handled by removing the degenerate components of the motion, therefore manipulator is made redundant to the task. Khatib's [3] dynamically consistent inverse was then used to invert the Jacobian and to create a null space motion to escape from the singular configuration in the case that the desired path lies in the degenerate direction. The algorithm was implemented on the PUMA 560 manipulator, and the results are presented.
\end{abstract}

\section{Introduction}

Conventional control algorithms of a robot manipulator are often found to use the solution of inverse kinematics or inverse Jacobian to transform various tasks into desired joint motions. These methods were found to be insufficient in the vicinity of a singular configuration, as they produce high joint velocity and large error in end-effector's trajectory.

Various ways have been devised to handle the problem of singularity, starting from the simple approach of switching into joint space control. Others try to avoid going near the singular configuration by maximizing the manipulability of the end-effector at all time [7] [11]. However, this is not always possible when the manipulator is not redundant with respect to the task.

Another idea in handling singularity was to eliminate the degenerate component(s) of motion and therefore, there will be no large joint velocity generated when the manipulator goes into the vicinity of singularity [3] [4] [5]. Chiaverini and Egeland [5] used partial Jacobian matrix, with rows along the singular direction eliminated, was inverted using pseudo inverse to obtain joint velocity from velocity in task space. Aboaf and Paul [1] bounded the rates of excessive joints and compensated for the error by a modified inverse Jacobian to 
calculate the remaining joint rates. Cheng et al [4] analyzed the singularities in PUMA and decomposed the workspace into achievable and non-achievable directions. The non-achievable direction was released, Compact QP method was applied to minimize the tracking error of the end-effector, and SVD was used to obtain the Jacobian inverse and a weight system to regulate the trade off between exactness and feasibility of motion.

The experiment described in this report was conducted on the platform of Operational Space Formulation [9]. In this scheme, motion is generated not by kinematics-based method, but by the inertial parameters of the manipulator. The necessity of using the inverse of Jacobian comes from the calculation of the dynamic model of the manipulator. In comparison to the conventional method of kinematics-based control, singularity handling in Operational Space Formulation covers the problem both in motion and force control. The proposed idea is to handle singularity by transforming the Jacobian and the generalized forces into the frame whose one or more of the axes represent the degenerate direction(s) of singularity. The degenerate component is then removed resulting in a lower dimension, full rank Jacobian that is redundant with respect to the task. The dynamically consistent inverse [3], which is a generalised inverse weighted by inertial matrix, is used to invert the Jacobian. Null space motion [9] was also used to reconfigure the manipulator to escape from singularity into a path that happens to lie in the degenerate direction, which we have removed. The experimental result on PUMA 560 is presented.

\section{Operational Space Formulation}

Operational Space Formulation [9] was devised to enable a unified approach on the force and motion control of a manipulator. In this scheme, we would be able to resolve the singularity issue in both motion and force control. In the scope of this experiment, we are more concerned about the motion control part of the algorithm. Motion of the end-effector is generated by:

$$
\Gamma=J^{T} F+N^{T} \Gamma_{0}
$$

where $\Gamma$ is the joint torque, $\mathrm{J}$ is the Jacobian matrix, and $\mathrm{F}$ is the desired force obtained by:

$$
F=\hat{\Lambda} \ddot{x}+\hat{\mu}(x, \dot{x})+\hat{p}(x)
$$

where $\Lambda$ is the pseudo kinetic energy matrix, $\hat{\mu}(x, \dot{x})$ is the Coriolis and Centrifugal matrix, and $\hat{p}(x)$ is the gravity compensation matrix. $\mathrm{N}$ is the null space of the manipulator defined as $N=[I-\bar{J} J]$, and $\Gamma_{0}$ is the null space torque.

$\ddot{x}$ is the linear dynamic behaviour defined by:

$$
F^{*}=I \ddot{x}_{d}-k_{v}\left(\dot{x}-\dot{x}_{d}\right)-k_{p}\left(x-x_{d}\right)
$$

where $\ddot{x}_{d}, \dot{x}_{d}, x_{d}$ are the desired acceleration, velocity, and position of the endeffector at the given time, and $\ddot{x}, \dot{x}, x$ are the actual acceleration, velocity, and position of the end-effector. It should be noted, that throughout this experiment, the task given would be to control both position and orientation in $3 \mathrm{D}$ space (6 DOF task in motion control). 


\section{Dynamic Formulation}

The need for inverse of the Jacobian in the dynamic formulation comes from the derivation of the $\Lambda, \mu$, and $p$ matrices. These are the pseudo kinetic energy, Coriolis, and Centrifugal matrices in task space, and were all derived from the joint space equivalent. They are derived in [9] and [10] as the following:

$$
\begin{gathered}
\Lambda(x)=J^{-T}(q) A(q) J^{-1}(q) \\
\mu(x \dot{x})=\left[J^{-T}(q) B(q)-\Lambda(q) H(q)\right][\dot{q} \dot{q}] \\
p(x)=J^{-T}(q) g(q)
\end{gathered}
$$

where $\mathrm{A}(\mathrm{q})$ is the kinetic energy matrix, $\mathrm{B}(\mathrm{q})$ and $\mathrm{H}(\mathrm{q})$ are matrices of the Coriolis and Centrifugal forces, and $\mathrm{g}(\mathrm{q})$ the gravitational compensation matrix.

For further explanation of the Operational Space Formulation, please refer to $[8],[9]$ and $[10]$.

\section{Singularity Identification in PUMA}

Singularity is defined as the configuration where the values of joint position causes the Jacobian to become singular (the determinant of the Jacobian is zero) or when the manipulator loses degree(s) of freedom [11]. The degenerate direction is defined as the degree of freedom that the manipulator loses in the singularity, or a motion that the manipulator can not execute while in singularity. It is also represented by the component of the Jacobian matrix that causes it to be singular.

Khatib [10] categorizes singularities into two types. Type 1 is when null space torque creates motion in the degenerate direction. Type 2 is when null space torques affects only the internal joint motions, or when the null space motion moves (shifts) the degenerate direction.

In PUMA, or many PUMA-like manipulators (or referred to as anthropomorphic with spherical wrist manipulator in [12]), there are three singularities: wrist lock (type 2), elbow lock (type 1), and head lock (type 2). These singularities can happen individually, or as a combination of two of even three at the same time.

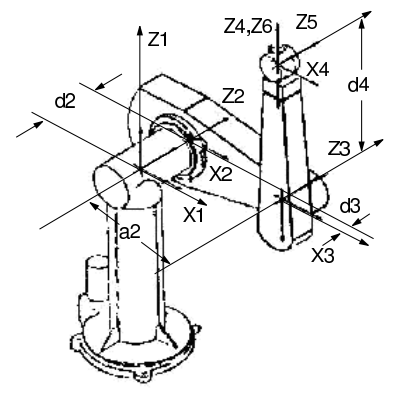

Figure 1. The PUMA 560 at zero position, by Craig's modified DH parameter $[6]$ 
Table 1. THE MODIFIED DH PARAMETERS

\begin{tabular}{ccccc}
\hline $\mathrm{i}$ & $\alpha_{i-1}$ & $a_{i-1}$ & $d_{i}$ & $\vartheta_{i}$ \\
\hline 1 & 0 & 0 & 0 & $\vartheta_{1}$ \\
2 & -90 & 0 & $d_{2}$ & $\vartheta_{2}$ \\
3 & 0 & $a_{2}$ & $d_{3}$ & $\vartheta_{3}$ \\
4 & 90 & $a_{3}$ & $d_{4}$ & $\vartheta_{4}$ \\
5 & -90 & 0 & 0 & $\vartheta_{5}$ \\
6 & 90 & 0 & 0 & $\vartheta_{6}$ \\
\hline
\end{tabular}

Mathematically, singularity occurs when the determinant of the Jacobian matrix approaches zero, i.e.: $\operatorname{Det}(J)=0$ or $\operatorname{Det}\left(J J^{T}=0\right)$ for manipulators with non-square Jacobians [11].

For PUMA 560 with 6 DOF, the Jacobian is a square $6 \times 6$ matrix, which can be partitioned into:

$$
J=\left[\begin{array}{ll}
J_{11} & J_{12} \\
J_{21} & J_{22}
\end{array}\right]
$$

By defining the control point to be at the wrist, we will obtain a Jacobian matrix with $J_{12}=0_{3 \times 3}$. With the frame assignment shown in Figure 1, and modified DH parameters according to Craig's [6] (See Table 1), the determinant of PUMA is shown as:

$$
\begin{gathered}
\operatorname{Det}(J)=\operatorname{Det}\left(J_{d}\right)=\operatorname{Det}\left(J_{11}\right) \operatorname{Det}\left(J_{22}\right) \\
\operatorname{Det}\left(J_{11}\right)=-a_{2}\left(d_{4} C_{3}-a_{3} S_{3}\right)\left(d_{4} S_{2} 3+a_{2} C_{2}+a_{3} C_{2} 3\right) ; \\
\operatorname{Det}\left(J_{22}\right)=-S_{5}
\end{gathered}
$$

(Cheng et al[4])

where $a_{2}=0.4318(m), a_{3}=-0.0203(m), d_{2}=0.2435(m)$, $d_{3}=-0.0934(m), d_{4}=0.4331(m)$.

When the determinant equals zero, Equation 6 represents the elbow, head, and wrist singularities respectively.

\section{Identifying the Singular Direction}

When singularity occurs, there is a row(s) in the Jacobian - when it is transformed onto the correct frame - that contains only zeros. By definition that $\dot{x}=J \dot{\vartheta}$, a zero row in the Jacobian means that there is a direction (or a degree of freedom) in task space that the manipulator is unable to move into. This is the case in singular configuration, as the manipulator loses a degree of freedom at these points.

When Head Lock occurs, $a_{2} \cdot C_{2}+a_{3} \cdot C_{23}+d_{4} \cdot S_{23}=0$ (Equation 6). Equation(7) shows the top half of the Jacobian ${ }^{4} J$. From this equation, it is shown that the second row of the Jacobian is zero, which corresponds to the translation along $\mathrm{Y}$-axis of Frame $\{1\}$ which is the degenerate direction.

$$
{ }^{1} J=\left[\begin{array}{cccccc}
-d_{2}-d_{3} & d_{4} C_{23}-a_{2} S_{2}-a_{3} S_{23} & d_{4} C_{23}-a_{3} S_{23} & 0 & 0 & 0 \\
a_{2} C_{2}+a_{3} C_{23}+d_{4} S_{23} & -a_{2} C_{2}-a_{3} C_{23}-d_{4} S_{23} & -a_{3} S_{23}-d_{4} S_{23} & 0 & 0 & 0 \\
0 & 0 & 0 & 0
\end{array}\right]
$$

For the Wrist Lock, there is no entire row of zeros in the $\mathrm{J}$ matrix (Equation 
(8))

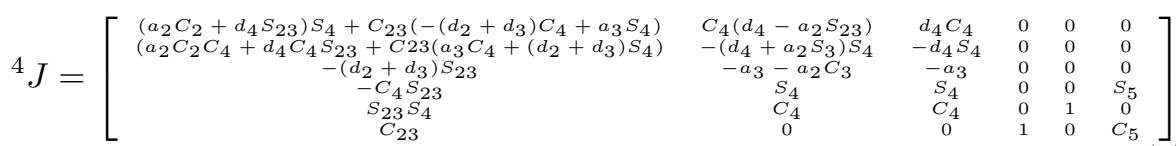

When $\left(\vartheta_{5}=0\right)$, a row of zero only appears at the last three elements of the fourth row of ${ }^{4} J$ :

$$
{ }^{4} J_{22}=\left[\begin{array}{ccc}
0 & 0 & 0 \\
0 & 1 & 0 \\
1 & 0 & 1
\end{array}\right]
$$

This means that it is still possible to rotate around the X-axis of Frame $\{4\}$ in wrist singularity, but it is produced by the first three joints, which would also change the position of the end effector (i.e. not possible in 6 DOF).

Therefore, the first row of ${ }^{4} J_{22}$, (or the fourth row of ${ }^{4} \mathrm{~J}$ ) is the degenerate direction, representing the rotation around X-axis of Frame $\{4\}$ (see Figure 1 and 4). Collapsing the Jacobian is then done by eliminating the fourth row of ${ }^{4} J$.

Elbow singularity is shown by projecting the Jacobian and the task space forces onto Frame $\{\mathrm{B}\}$ which is not one of the frames in our DH assignment (see Figure 2 for the frame assignment, and Equation (10) for the resulting Jacobian). At this configuration, the singular direction is found to fall along the line connecting the wrist point to the origin of base frame.

${ }^{B} J[1][1]=\frac{\left(d_{2}+d_{3}\right) S_{2}\left(d_{4} C_{3}-a_{3} S_{3}\right)}{\left(a_{2}+a_{3} C_{3}+d_{4} S_{3} D\right.}$

${ }^{B} J[1][2]=\frac{\left(d_{4} C_{3}-a_{3} S_{3}\right)\left(a_{2}+a_{3} C_{3}+d_{4} S_{3}\right)^{2} D}{a^{2}+d^{2}+2 d_{2} d_{3}+d^{2}+a_{2} a_{3}+a_{3} C_{3}^{2}+2 a^{2} d_{4}+d_{2} S_{3}+a}$

${ }^{B} J[1][3]=\frac{d_{4} C_{3}-a_{3} S_{3}}{D}$

${ }^{B} J[2][1]=\left(d_{2}+d_{3}\right) S_{2} ;{ }^{B} J[2][2]=a_{2}+a_{3} C_{3}+d_{4} S_{3}$

${ }^{B} J[2][3]=a_{3} C_{3}+d_{4} S_{3}$

${ }^{B} J[3][1]=\frac{C_{2}\left(a_{2}^{2}+d_{2}^{2}+2 d_{2} d_{3}+d_{3}^{2}+a_{2} a_{3} C_{3}+a_{2} d_{4} S_{3}\right)+\left(a_{2}+a_{3} C_{3}+d_{4} S_{3}\right)\left(a_{3} C_{23}+d_{4} S_{23}\right)}{\left(a_{2}+a_{3} C_{3}+d_{4} S_{3}\right) D}$

${ }^{B} J[3][2]=\frac{\left(d_{2}+d_{3}\right)\left(d_{4} C_{3}-a_{3} S_{3}\right)}{\left(a_{2}+a_{3} C_{3}+d_{4} S_{3}\right) D} ;{ }^{B} J[3][3]=\frac{\left(d_{2}+d_{3}\right)\left(d_{4} C_{3}-a_{3} S_{3}\right)}{\left(a_{2}+a_{3} C_{3}+d_{4} S_{3}\right) D}$

where:

$$
D=\sqrt{1+\frac{\left(d_{2}+d_{3}\right)^{2}}{\left(a_{2}+a_{3} C_{3}+d_{4} S_{3}\right)^{2}}}
$$

Frame $\{\mathrm{B}\}$ is obtained by rotating Frame $\{2\}$ by angle $\beta$, which is defined as:

$$
\beta=\operatorname{Tan}^{-1}\left[\frac{d_{2}+d_{3}}{a_{2}+a_{3} C_{3}+d_{4} S_{3}}\right]
$$

From (6), it is shown that $-a_{2}\left(d_{4} C_{3}-a_{3} S_{3}\right)=0$ at elbow singularity. Therefore, the first row of ${ }^{B} J_{11}$ is a zero row $\left({ }^{B} J[1][1]={ }^{B} J[1][2]={ }^{B} J[1][3]=0\right)$. This shows that the degenerate direction lies along the $\mathrm{X}$-axis of Frame $\{\mathrm{B}\}$ (see Figure 2). Equation 10 only shows the elements ${ }^{B} J$ from the top left quadrant, because the top right quadrant is a zero matrix.

\section{Removing the Degenerate Component}

The idea of removing the degenerate component is done by removing the row $(\mathrm{s})$ of the Jacobian matrix and elements of the task space force F (see Equation (2)) 


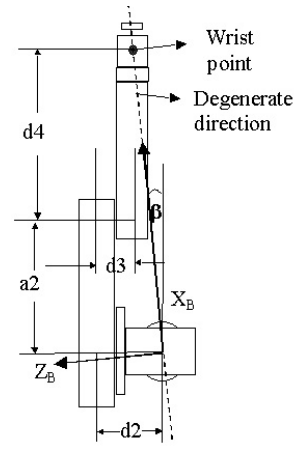

Figure 2. PUMA, from top view, shows the degenerate direction at elbow singularity, expressed in Frame $\{B\}$, which is derived from rotating Frame $\{2\}$ by angle $\beta$

that represent the degenerate direction(s) of motion. To do so, the Jacobian matrix and force vector need to be expressed in the frame in which one of the axes represents the direction of singularity (degenerate direction).

Force vector in task space is obtained from the control law to represent the virtual force that 'pulls' the end effector to the desired position and orientation (see Equations (2), (3)). The force vector is then resolved into the correct frame, that represents the singular direction as one of the axes of the frame. The Jacobian is also resolved into the same frame, and the degenerate components in the Jacobian and the force vector are removed. The transpose of the new collapsed Jacobian and the force vector are multiplied to obtain the joint torque vector (Equation (1)).

For the case of PUMA 560, the degenerate direction of motion in wrist singularity, for example, is represented by the rotation around the $\mathrm{X}$-axis of Frame $\{4\}$. The relationship between the generalized forces, expressed in the frame that best represent the singular direction, is therefore (without the Null Space component:

$$
\Gamma={ }^{4} J^{T 4} F
$$

where

$$
{ }^{4} J^{T}={ }^{0} R_{4}^{T^{4}} J \text { and } \quad{ }^{4} F={ }^{0} R_{4}^{T^{4}} J
$$

${ }^{4} F$ is the force in the Cartesian axis represented in Frame\{4\}, obtained from the control law with the fourth element (rotation around $\mathrm{X}$-axis) removed, $\Gamma$ is the torque sent out to each joint, and ${ }^{4} J$ is the Jacobian expressed in Frame $\{4\}$, with the fourth row removed.

\section{Moving Through Singularities}

Motion from a singular configuration can be divided into of feasible and nonfeasible directions. Non-feasible direction is one that requires motion in the singular (degenerate) direction.

In our experiment, as explained above, the motion in the degenerate direction has been disabled through the removal of the elements of the Jacobian 
matrix and task space forces representing the direction of singularity.

In the case of a feasible path, the end-effector was found to move through the singular configuration with error in position and orientation not significantly larger than that of motion through non-singular configuration. (Compare Figures A1 and A2 which represent tracking error in non-singular motion, with Figures A3 and A4 which represent tracking error of motion through a wrist singularity along a feasible path).

A path is non-feasible when the desired trajectory lies along the degenerate direction of the manipulator. In this experiment, the null space motion was then utilized in handling such motion. As the Jacobian was collapsed to be of lower rank, the manipulator is now regarded as 'redundant' with respect to the task. Null space torque can then be generated to reconfigure the manipulator to move in the non-feasible direction. Different potential functions can be used to determine the Null space torque, as long as the objective is to shift the degenerate direction out of the way of the desired trajectory.

Khatib [9] showed that the relationship between the generalized forces incorporating the null space torque, as in Equation 1. The $\bar{J}$ is the inverse of the Jacobian matrix, obtained by:

$$
\bar{J}=A^{-1}(q) J^{T}(q) \Lambda(q)
$$

where the components are defined in (4).

This is described as the dynamically consistent inverse [3], which is a generalised inverse weighted with mass (inertial) matrix. This inverse has been shown to produce no operational space acceleration when used to project joint torques into the null space[3].

The following sections show the result of the implementation the algorithm on PUMA 560. It is shown that in most cases, the error generated is not larger than that found in non-singular motion, except for some trade off shown in the cases of desired trajectory lying along non-feasible path, where exactness of orientation tracking was sacrificed to make the motion feasible.

\subsection{Type 1 Singularity}

Type 1 Singularity, or in the case of PUMA, the elbow lock, is one where null space torque would generate motion in the singular direction.

This means, for the case of PUMA, null space motion of joint 3 would generate motion in the singular direction (see Figure 2 for singular direction). Comparing the tracking error (position and orientation) of the manipulator moving out of elbow singularity into the degenerate direction with that of non-singular motion, no significant increase in position and orientation error is observed. (Compare Figure A7 and A8 to Figure A1 and A2).

\subsection{Type 2 Singularity}

A common type 2 singularity in PUMA is when the wrist joint is straightened $\left(\vartheta_{5}=0\right)$. The non-feasible path is when it contains the component of degenerate direction, i.e. it requires the end-effector to turn around the $\mathrm{X}$-axis of Frame $\{4\}$, or if the desired trajectory lies on the YZ plane of Frame $\{4\}$. The other one is the head lock, where the wrist point lies along the $\mathrm{Z}$ axis of 


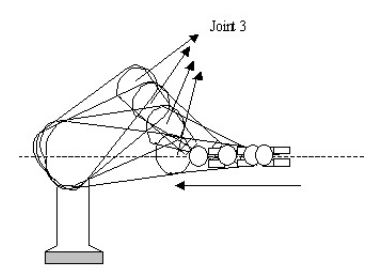

Figure 3. shows Puma-like manipulator moving out of elbow (and wrist) singularity, following the path which lies in the degenerate direction

the Base Frame. The degenerate direction is the Y-axis of Frame $\{1\}$. It is described that, as singularity occurs, at least two frames of the manipulator would line up. One of these two frames can therefore be used to create a null space motion to escape the singular position. In the case of PUMA's wrist, null space torque was used to move joint 4 (see Figure 4). This would then shift the plane that contains the non-feasible-path (the YZ plane) out of the way of the desired path (Figure 5). Similarly, in the case of PUMA head lock,

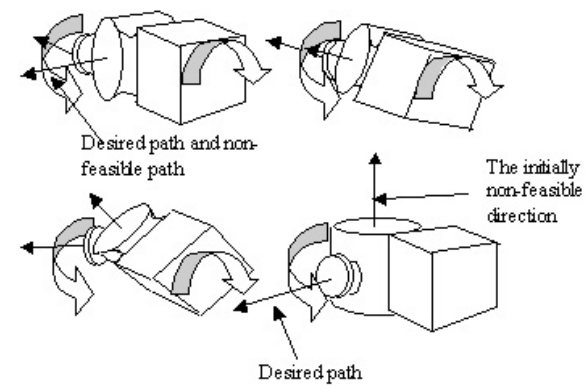

Figure 4. Null space torque is used to turn joint 4, so that the YZ plane of Frame $\{4\}$ is shifted out of the way of the desired trajectory.

a null space motion of joint 1 is required to shift the the degenerate direction out of the desired path. The result of the experiment is shown in Figures A1 to A8. Figures A1 and A2 show the position and orientation error in end-effector tracking a non-singular path, while Figure A3 and A4, a singular, but feasible path. Figure A5 and A6 is of the end-effector escaping the wrist lock (type 2 singularity) into non-feasible path, and Figure A7 and A8 of the end-effector moving from an elbow singularity into a non-feasible path. The only significantly larger error encountered is in the orientation error of the manipulator as it escapes from a wrist lock into a path that lies in degenerate direction. It is because null space motion was required to turn joint 4 before the end-effector was able to track the desired trajectory, hence the large initial orientation error (see Figure 4). 


\section{Implementation Issues}

Jerkiness in due to a switching of control was experienced as expected. This is especially obvious when the manipulator moves in the degenerate direction by null space torque to escape elbow singularity as in this motion it is not motion-controlled. It therefore does not follow the desired trajectory while in the singular region.

The chosen solution in the experiment was to take the end effector at the point (time and position) of getting out of the singular region as the start of a new trajectory, with the same goal as before, compensating for the time it took for the manipulator to reach this point from the starting point. This was found to produce a smooth transition between the control algorithms.

\section{Conclusion}

In this experiment, singularity handling in torque-controlled manipulator, based on Operation Space Formulation was explored. It was shown that by removing the degenerate component(s) of motion, control of the manipulator through singular configuration was possible, with tracking error no larger than that of a motion through non-singular path. Motion in the degenerate direction was made possible by motion of redundant joint to move the degenerate direction away from the desired path for type 2 singularities. For type 1 singularity, by definition, Null space torque would produce motion in the degenerate direc-

tion. A certain trade off between exactness and achievability was necessary in moving out of a singular configuration into a non-feasible path.

\section{Acknowledgement}

The authors would like to acknowledge and to thank Professor Oussama Khatib for his guidance in his capacity as the advisor to the project.

\section{References}

[1] E.W. Aboaf and R.P. Paul 1987 Living with the Singularity of Robot Wrists. IEEE Intl. Conf. for Robotics and Automation pp 1713-1717.

[2] B. Armstrong, O. Khatib, J. Burdick 1986 The Explicit Dynamic Model and Inertial Parameters of the PUMA 560 Arm.IEEE Intl. Conf. Robotics and Automation pp 510-518.

[3] K. Chang and O. Khatib 1995 Manipulator Control at Kinematic Singularities: A dynamically consistent Strategy. Proc. IEEE/RSJ Int. Conference on Intelligent Robots and Systems Pittsburgh, vol. 3, pp. 84-88.

[4] F.T. Cheng et al 1997 Study and Resolution of Singularities for a 6-DOF PUMA Manipulator. IEEE Trans. Systems, Man and Cybernetics Part B, vol.27 2, pp:332343.

[5] S. Chiaverini and O. Egeland 1990 A Solution to the Singularity Problem for Six-joint Manipulators. Proc. IEEE for Robotics and Automation vol 1 pp 644-649.

[6] John J. Craig 1989 Introduction to Robotics, Mechanics and Control. 2nd ed, Addison-Wesley.

[7] P. Hsu, J. Hauser, S. Sastry 1988 Dynamic Control of Redundant Manipulators. IEEE Int; Conf. Robotics and Automation vol. 1, pp 183-187. 
[8] Jamisola, R., Ang, M, Jr., Lim, T.M., Khatib, O., Lim, S.Y.1999 Dynamics Identification and Control of an Industrial Robot. The Ninth Intl. Conf. On Advanced Robotics pp 323-328.

[9] O.Khatib 1987 A Unified Approach for Motion and Force Control of Robot Manipulators: The Operational Space Formulation. IEEE J. Robotics and Automation vol. RA-3, no. 1 , pp 43-53.

[10] O.Khatib 1996 Advanced Robotics Lecture Notes, Stanford University.

[11] Y. Nakamura 1991 Advanced Robotics - Redundancy and Optimization AddisonWesley.

[12] L. Sciavicco and B. Siciliano 1990 Modeling and Control of Robot Manipulators McGraw-Hill.

\section{Appendix: Experimental Results}

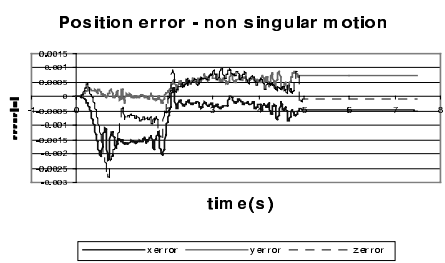

Figure A1.

Position error - w rist lock - feasible path

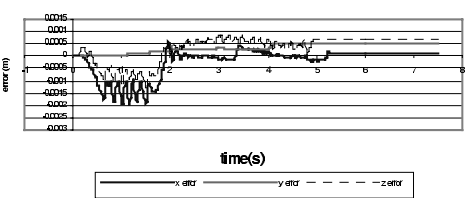

Figure A3.

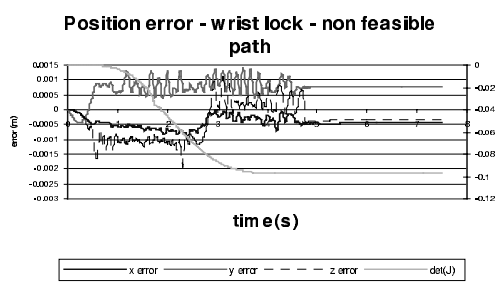

Figure A5.

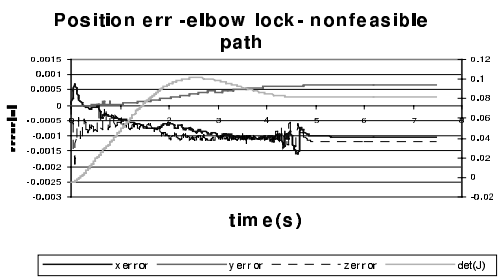

Figure A7.
Orientation error - non-singular motion

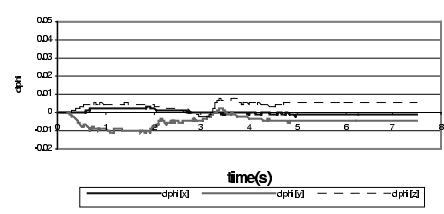

Figure A2.

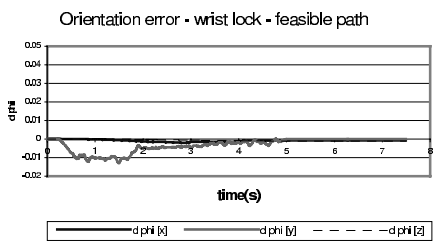

Figure A4.

Orientation error - wrist lock - nonfeasible path

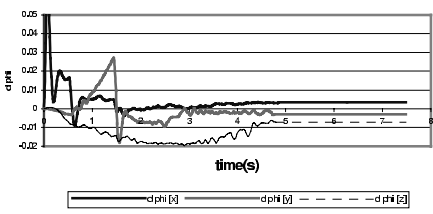

Figure A6.

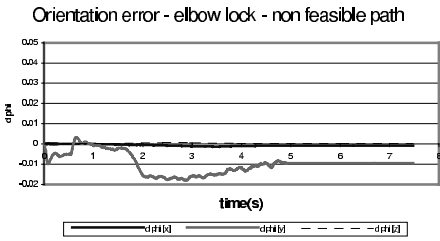

Figure A8. 